AperTO - Archivio Istituzionale Open Access dell'Università di Torino

Probing the Mechanochemistry of Metal-Organic Frameworks with Low-Frequency Vibrational Spectroscopy

This is a pre print version of the following article:

Original Citation:

Availability:

This version is available http://hdl.handle.net/2318/1685824

since 2019-01-07T11:52:21Z

Published version:

DOI:10.1021/acs.jpcc.8b08334

Terms of use:

Open Access

Anyone can freely access the full text of works made available as "Open Access". Works made available under a Creative Commons license can be used according to the terms and conditions of said license. Use of all other works requires consent of the right holder (author or publisher) if not exempted from copyright protection by the applicable law. 


\section{Probing the Mechanochemistry of}

\section{Metal-Organic Frameworks with}

\section{Low-Frequency Vibrational Spectroscopy}

Wei Zhang, ${ }^{\dagger, \|}$ Jefferson Maul, ${ }^{\ddagger} \|$ Diana Vulpe, ${ }^{\Uparrow}$ Peyman Z. Moghadam, ${ }^{\S}$ David

Fairen-Jimenez, "Daniel M. Mittleman, ${ }^{\dagger}$ J. Axel Zeitler, Alessandro Erba, ${ }^{*} \ddagger$ and Michael T. Ruggiero* $\boldsymbol{\top}, \perp$

$\dagger$ School of Engineering, Brown University, Providence, RI, 02912, United States of America

$\ddagger$ Dipartimento di Chimica, Universitá di Torino, via Giuria 5, 10125, Torino, Italy

\Department of Chemical Engineering and Biotechnology, University of Cambridge, Philippa Fawcett Drive, Cambridge, CB3 0AS, United Kingdom

$\S$ Department of Chemical and Biological Engineering, University of Sheffield, Mappin

Street, Sheffield, S1 3JD, United Kingdom

॥Equal Contribution

$\perp$ Department of Chemistry, University of Vermont, 82 University Place, Burlington, VT 05405, United States of America

E-mail: alessandro.erba@unito.it; michael.ruggiero@uvm.edu

Phone: +44 (0) 1223334783 


\begin{abstract}
The identification and characterization of low-frequency vibrational motions of metalorganic frameworks (MOFs) allows for a better understanding of their mechanical and structural response upon perturbation by external stimuli such as temperature, pressure, and adsorption. Here, we describe the combination of an experimental temperatureand pressure-dependent terahertz spectroscopy system with quantum mechanical simulations to measure and assign specific low-frequency vibrational modes that directly drive the mechanochemical properties of this important class of porous materials. More specifically, those intense spectral features in the terahertz region of the vibrational spectrum of ZIF-8 are identified, which are directly connected to its mechanochemical response. In particular, the mechanical compressibility of pristine ZIF-8 is found to follow a peculiar non-linear trend upon pressure: its bulk modulus initially increases up to $0.1 \mathrm{GPa}$ and decreases at higher pressures, which is simultaneously reflected in the terahertz vibrational spectra. This work highlights the interplay between structural, vibrational, and mechanochemical phenomena, all of which are key to the effective exploitation of MOFs. The importance of terahertz vibrational motions on the function of MOFs is demonstrated, and a method presented for their measurement and interpretation, which can be applied widely to any supramolecular material.
\end{abstract}




\section{Introduction}

The connections between low-frequency large-amplitude terahertz vibrations and material function are becoming increasingly apparent. These motions have been shown to play a critical role in enzymatic catalysis and ligand binding, vibrational dampening and relaxation, crystallization and amorphization phenomena, phase transitions, structural response to temperature and pressure (e.g. negative thermal expansion), etc. ${ }^{1-7}$ Recently, Ryder et al. have analyzed the low-frequency lattice dynamics of metal-organic frameworks (MOFs) and suggested that they can play an important role into their proper function and behavior; however no explicit connection between these phenomena has been established beyond inference (based on spectral assignment). ${ }^{8,9}$ In this respect, the current challenge is that of establishing an explicit link between specific terahertz lattice vibrations and specific MOF functionalities, which would increase their effective design and use.

MOFs are a promising class of porous materials owing to their intrinsic structural and mechanical properties and large specific surface areas. They have been proposed for industrial applications such as energy storage, drug delivery, catalysis and chemical separation. ${ }^{10-15}$ MOFs can be readily tuned through the modification of either the metal cluster or organic linkers, leading to a virtually infinite number of materials that are yet to be fully explored. ${ }^{16,17}$ The diversity found in MOFs brings a unique combination of structural, chemical, and mechanical properties that play a critical role in their behavior and performance. So far, little attention has been paid to the role that the nuclear dynamics, particularly the low-frequency dynamics, has on these phenomena.

Among the existing MOFs that can be studied, ZIF-8 (ZIF: zeolitic imidazolate framework) is a prototypical example that has been widely investigated, for which two crystalline

phases and an amorphous phase have been reported. ${ }^{18-20}$ Importantly, these two crystalline phases are characterized by a critical change in pore size and accessibility. Moggach et al. described a first-order adsorption-induced phase transition at 1.47 GPa when using methanol as a pressure-transmitting medium (PTM) under hydrostatic conditions; ${ }^{20}$ when using a non- 
penetrating fluid as the PTM (i.e. no guest molecules in the cavities) a pressure-induced amorphization was observed at $0.34 \mathrm{GPa} .{ }^{19}$ In recent years, a number of studies, both experimental and theoretical, have been performed to study the role of pressure, temperature, and the nature of the gas molecules on the adsorption capacity of ZIF-8, including the apparently counterintuitive case of an increased pore window size upon pressure. ${ }^{20-25}$ More specifically, using both in situ powder X-ray diffraction and molecular simulation, it has been shown that a "gate-opening" or "swing-effect" adsorption-induced structural change occurs in ZIF8 at much lower pressures, where - similar to the $1.47 \mathrm{GPa}$ phase described above - the imidazolate linkers rotate so as to increase the size of the accessible pore window. ${ }^{21,26,27}$ These classes of motions, i.e. large-amplitude displacements of entire molecular sub-units, occur at terahertz frequencies, ${ }^{28}$ confirming that terahertz vibrations play a key role in the mechanochemical properties and phase-transition pathways present in such solids.

Low-frequency vibrational spectroscopies have been used to study ZIFs, ${ }^{9,29}$ providing valuable information related to the specific vibrational motions that are present, as well as for the detection of gas-adsorption in ZIF-8. ${ }^{30}$ Terahertz time-domain spectroscopy (THzTDS) can be used to acquire experimental spectra of infrared active vibrational modes in the far-infrared. Compared to traditional far-infrared spectroscopy, the method is convenient in that it can collect high quality spectra within seconds with signal-to-noise characteristics far exceeding that of Fourier transform instruments. ${ }^{31}$ THz-TDS is particularly useful for the investigation of ZIFs because it directly probes large-amplitude collective atomic motions that are expected in the terahertz frequency range $\left(0.1-10 \mathrm{THz}, 3-333 \mathrm{~cm}^{-1}\right)$. These vibrations are strongly dependent on both the intermolecular and intramolecular potential energy landscape, and thus THz-TDS is a sensitive probe of all the interactions present within solids. While the relationship between lattice dynamics and mechanochemical phenomena has been clearly long established, ${ }^{32,33}$ recent work in the MOF community has focused on identifying specific low-frequency vibrational modes that might be relevant to the elastic response of such materials. ${ }^{8,9}$ For the MOF community these exploratory studies have very usefully 
directed attention towards considering the well-known relationship between the terahertz (lattice) vibrations and mechanical properties. However, given the proof-of-concept nature of those studies, the conclusions drawn were necessarily based on the qualitative inspection of the vibrational mode types, with emphasis on motions that were considered to involve pathways corresponding to particular deformations (e.g. shear deformation). A quantitative analysis of the mechanisms was not possible as the experimental data was largely based on inelastic neutron scattering (INS) and synchrotron far-infrared measurements, techniques that, in contrast to THz-TDS, unfortunately exhibit a dramatic reduction in the signal-tonoise ratio at the low-frequencies $\left(<100 \mathrm{~cm}^{-1}\right)$, data which is critical to obtain quantitative insight.

Here, we discuss the interplay between pressure-induced structural, dynamical, and mechanical intrinsic features of a relatively flexible MOF: ZIF-8. In particular, we establish the relationship between specific low-frequency vibrational modes and the mechanochemical response of the material in the presence of external perturbations.

We propose a combination of experimental pressure- and temperature-dependent $\mathrm{THz}$ TDS and quantum-mechanical simulations to provide a consistent description of ZIF-8 upon compression. A peculiar non-linear pressure-dependence of the bulk modulus of ZIF-8 before amorphization is reported. We also describe the use of an innovative way to probe the effects of pressure and adsorption on the structure and dynamics of MOF systems, specifically when using gases that will adsorb, by combining in situ THz-TDS with a pressurized sample cell. Although we focus on ZIF-8, this approach can be extended to other MOFs and supramolecular materials in general. 


\section{Materials and Methods}

\section{Quantum-Mechanical Simulations}

\section{Solid-State Density Functional Theory}

The CRYSTAL17 software package was used for all solid-state density-functional-theory calculations. ${ }^{34}$ All simulations utilized the M06-2X density functional ${ }^{35}$ coupled with the allelectron double- $\zeta$ 6-31G(d,p) basis set due to previous success. ${ }^{29}$ Atomic positions were initialized from the experimentally determined single-crystal X-ray diffraction structure ${ }^{36}$ and were optimized at several pressures without any symmetry constraints by adding a diagonal hydrostatic pre-stress to the analytical stress tensor. ${ }^{37}$ It is important to note that due to ZIF-8 undergoing a phase-transition within the same space group $(I \overline{4} 3 m \rightarrow I \overline{4} 3 m)$ when using a penetrating PTM, identical results were obtained when performing simulations within the constraint of space group symmetry, as well as without any symmetry. Vibrational simulations were performed within the harmonic approximation via numerical differentiation (with atomic displacements of $0.003 \AA$ ),${ }^{38}$ and IR intensities were determined using the Berry Phase method. Energy convergence criteria were set to $\Delta E \leq 10^{-10}$ Hartree for both optimizations and frequency calculations. A shrinking factor of 4 is used for all calculations, corresponding to a $4 \times 4 \times 4$ mesh of $\mathbf{k}$ points and to 36 or $8 \mathbf{k}$ points in the symmetry-irreducible Brillouin zone without or with exploitation of the space group symmetry, respectively, and convergence was checked by performing single-point energy calculations at various $\mathbf{k}$ point samplings.

\section{Ab initio Molecular Dynamics}

The open-source CP2K software package was used for all AIMD simulations. ${ }^{39}$ Initially, 48 nitrogen molecules were randomly placed within the pore of the ZIF-8 crystal based on a minimum van der Waals radii method. Subsequently, both the loaded and unloaded crystals were simulated within the canonical (NVT) ensemble and the temperature was maintained 
at $100 \mathrm{~K}$ using a Nosé-Hoover chain thermostat. ${ }^{40}$ Unfortunately, CP2K does not have the meta-GGA functionals available, therefore the Perdew-Burke-Ernzerhof (PBE) density functional $^{41}$ was coupled with the double- $\zeta$ DZVP basis set ${ }^{42}$ and Goedecker-Teter-Hutter (GTH) pseudopotentials, ${ }^{43}$ with the D3-dispersion correction (with the Becke-Johnson damping functional) applied to all models. ${ }^{44}$ The energy convergence criterion was set to be $\Delta E<$ $10^{-9}$ hartree. The systems were equilibrated for 10 ps prior to performing the simulations for

vibrational analysis. IR spectra were generated by taking the Fourier transform of the dipole moment autocorrelation function, and all post-processing was performed using the TRAVIS software package. ${ }^{45}$ A 0.5 fs time step was used, and the molecular dipole moments were determined every 2.5 fs via determination of the Wannier centers. ${ }^{46}$ The spectra presented are obtained from a 30 ps trajectory, which has previously been shown to be sufficient for the vibrational analyses of molecular crystals. ${ }^{46}$

\section{Terahertz Time-Domain Spectroscopy}

30-35 mg of ZIF-8 sample were mixed with $360 \mathrm{mg}$ of high density polyethylene (PE) and compressed into tablets with diameters of $6 \mathrm{~mm}$ and thicknesses of $2.5 \mathrm{~mm}$, and then placed into a custom-made pressure cell. No apparent amorphization occurred during the sample preparation due to the observation of a clear crystalline THz-TDS spectrum, as opposed to the featureless absorption expected of an amorphous solid. High pressure compressed gases (methane and nitrogen) were guided into the pressure cell to pressurize the sample tablet, and a liquid nitrogen cold finger and an electric heater were used to accurately control the sample temperature. The details regarding the operation of the pressure cell were previously reported. ${ }^{47}$ For each scan, we fixed the pressure and down-scanned the temperature from $300 \mathrm{~K}$ with a rate of $0.47 \mathrm{~K} / \mathrm{min}$.

A custom $\mathrm{THz}$ time-domain spectrometer was used to record the time-domain waveforms of the $\mathrm{THz}$ radiation that transmitted through the sample. ${ }^{47}$ The time-domain waveforms were truncated with an asymmetric tapered cosine window with a length of approximately 
16 ps to keep only the main THz pulse, and then zero-padded and Fourier transformed to obtain the spectra. The spectrum of a pure PE tablet was also measured, as the reference. The frequencies and linewidths of the modes were obtained by fitting the spectra to the sum of two Gaussian lineshapes $e^{-\left(f-f_{i}\right) / 2 \sigma_{i}^{2}}$ on top of a quadratic background $a f^{2}+b$, where $f$ is the frequency, $f_{i}$ is the central frequency of the $i$-th mode, $\sqrt{2 \ln 2} \sigma_{i}$ is the half width at half maximum of the $i$-th mode, and $a$ and $b$ are coefficients for the quadratic background.

\section{Results and Discussion}

\section{Origin of Anomalous Low-Pressure Mechanical Properties}

The relationship between pressure-dependent properties and performance of porous materials is of critical importance in applications such as in gas-storage and sequestration materials. Taking ZIF-8 - with a cubic lattice $(I \overline{4} 3 m)$ - as an example of a flexible MOF, several seemingly anomalous pressure-dependent features have been reported in the last few years. This is including the observation of (i) increasing pore accessibility with pressure, ${ }^{20}$ (ii) a negative value for the pressure derivative of the bulk modulus $K\left(\right.$ i.e. $\left.K^{\prime}\right)$ in the low pressure regime - suggesting the softening of the mechanical response of the system upon pressure, ${ }^{19}$ and (iii) an intrinsic amorphization above pressures of $0.34 \mathrm{GPa}$ when using a non-penetrating fluid as a PTM. ${ }^{19}$ Although ZIF-8 has been widely studied in the past, most experimental studies upon pressure involve adsorption of guest molecules inside its channels when penetrating PTM are used, making it very challenging to decouple intrinsic pressure-dependent features of the pristine framework from adsorption-induced ones.

In order to elucidate the atomistic origins of these anomalous structural and mechanical features, we performed solid-state quantum-mechanical simulations within the density functional theory (DFT) with the CRYSTAL17 program. ${ }^{34}$ We first found the optimized lattice parameter of ZIF-8, $17.04 \AA$, in excellent agreement with the experimental value of $17.0 \AA .{ }^{19}$ Additionally, the computed bulk modulus $K$ at zero pressure, $8.9 \mathrm{GPa}$, is also 
in excellent agreement with the experimental value of $7.7 \mathrm{GPa}$, as measured by Brillouin scattering. ${ }^{48}$ We then performed pressure-constrained structural relaxations at 13 pressures in the range 0-0.8 GPa. Figure 1 shows the structure of ZIF-8, with the major structural features involved in the large-amplitude terahertz motions explicitly labeled, as well as the predicted evolution of volume $(V)$ and bulk modulus $(K)$, the latter of which was obtained from the volume-pressure relationship according to $K(P)=-V(P)(\partial P / \partial V)$. As pressure increases, two main structural changes occur. First, a rotation of the imidazolate linking bridges (the "swing-effect" motion), described by the angle $\varphi$. Secondly, the $\mathrm{ZnN}_{4}$ tetrahedra twist, described by the angle $\theta$.

Considering the volume-pressure relationship, Chapman et al. reported the intrinsic structural and mechanical features of pristine ZIF-8 at low-pressures using in situ X-ray diffraction and a non-penetrating fluid as PTM. ${ }^{19}$ The fitting of the experimentally measured data in the 0 - 0.34 GPa pressure range using a third-order Birch-Murnaghan equation-ofstate provided the anomalous negative value for $K^{\prime}$ of -4.6 . From a theoretical point of view, classical molecular dynamics simulations were unable to confirm this peculiar feature as they described a bulk modulus almost linearly increasing as a function of pressure in the whole 0-0.4 GPa pressure range and thus a positive value of $K^{\prime} .{ }^{24}$ By fitting our simulated volumepressure data, reported in Figure 1 (b), in the same pressure range (since experimentally pressures above $0.34 \mathrm{GPa}$ resulted in amorphization, which is not accounted for in our model) and to the same equation-of-state, we get a negative value of $K^{\prime}=-3.8$, in good agreement with the experimental value. A quantum-mechanical description over a classical one is needed in order to catch such a subtle, and yet fundamental, feature of the mechanical response of ZIF-8, despite such simulations being performed at an effective $0 \mathrm{~K}$ temperature.

Let us stress that $K^{\prime}$ is just an average parameter, which does not reflect the punctual mechanical response of the system at the various pressures. Here, we want to determine the explicit $K(P)$ relation. We perform a more accurate fitting of the pressure-volume data using a higher-than-third-order function (sixth-order polynomial). This fitting allowed us to 
(a)

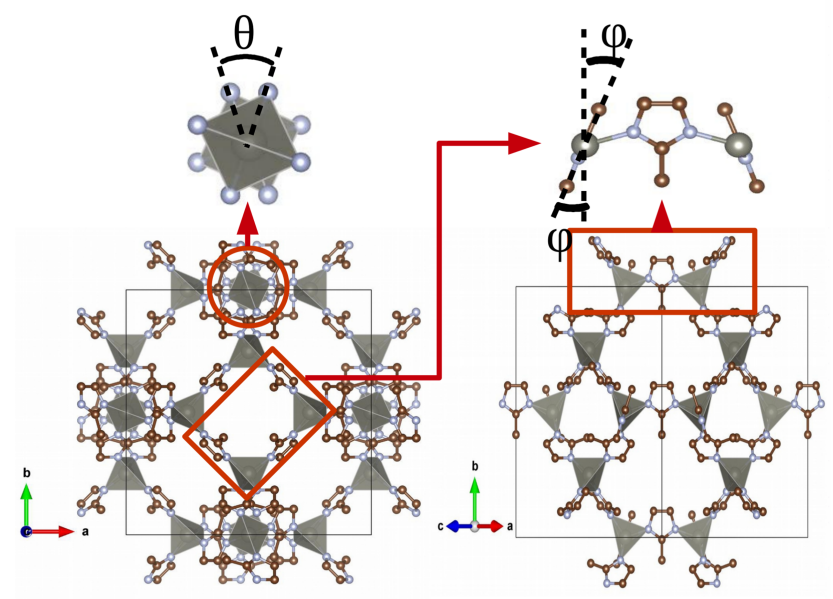

(b)

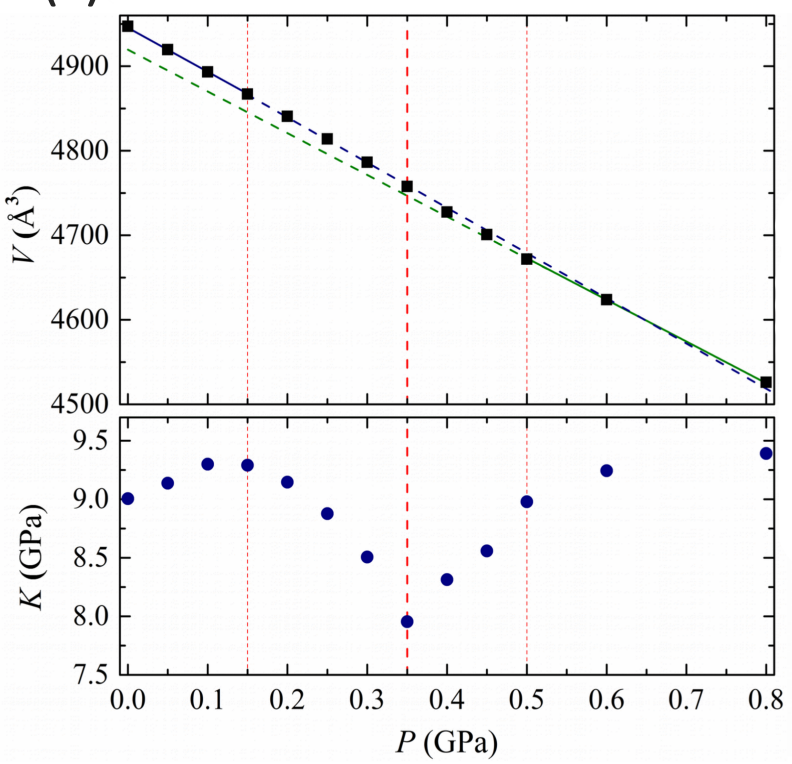

Figure 1: (a) Structure of ZIF-8; two angles $\varphi$ and $\theta$ are graphically defined, which represent the rotation of the imidazolate linkers and the twisting of the $\mathrm{ZnN}_{4}$ tetrahedra, respectively. (b) DFT-predicted volume $V$ and bulk modulus $K$ as a function of pressure in the low-pressure regime. Red dashed lines are eye-guides to identify three different structural trends, while the solid blue and green lines represent linear fits to the low and high-pressure regions, with dashed lines representing the extrapolated curves. 
investigate for the first time the explicit pressure dependence of the bulk modulus of ZIF-8 in that pressure range, which we report in Figure 1 (b). Three distinct regions are identified in the volume and bulk modulus: for $P<0.2 \mathrm{GPa}$ the $V(P)$ is almost linear, for $P>0.5$ GPa $V(P)$ is also almost linear, while for pressures $0.2<P<0.5 \mathrm{GPa}$, the $V(P)$ relation has a sigmoid-shape centered at $P=0.35 \mathrm{GPa}$ (i.e. a point where the $K(P)$ relation changes the sign of the slope). Regarding the mechanical properties of ZIF-8, we find that the bulk modulus initially increases up to about $0.1 \mathrm{GPa}$, then decreases above $0.2 \mathrm{GPa}$, eventually showing a clear discontinuity at $0.35 \mathrm{GPa}$. This matches the experimental evidence according to which pressurization beyond $0.34 \mathrm{GPa}$ produces irreversible structural changes leading to the pressure-induced amorphization of the sample possibly due to the disruption of longrange translational symmetry while retaining the short-range order (e.g. local structure and framework connectivity). ${ }^{19}$ Again, it is important to make clear that the amorphization observed experimentally is not captured by our model due to the imposition of translational symmetry that prohibits long-range structural disorder, however the data obtained allows for some insight into the properties of ZIF-8 when amorphization is restricted.

\section{Spectroscopic Fingerprint of Mechanical Softening on Compression}

Terahertz spectroscopy has been demonstrated to be an effective technique for probing relevant atomic motions in MOFs. ${ }^{8,9,29}$ However, the assignment of the low-frequency spectrum for the "swing-effect" dynamics in ZIF-8, with peaks at about $1.0 \mathrm{THz}$ (Raman active) and $2.0 \mathrm{THz}$ (infrared active), has been controversial. ${ }^{9,29}$ While a later study has claimed the spectral assignment by Tan et al. ${ }^{29}$ to be the correct one, ${ }^{30}$ the results of the present study clearly show that both previously identified peaks indeed correspond to the "swing-effect" motion of the imidazolate linkers (the peak at about $1 \mathrm{THz}$ being the symmetric "gate-opening" motion while the peak at about $2.0 \mathrm{THz}$ being the asymmetric "gate-opening" motion). Crucially, this study allows for an explicit correlation of these spectral features with structural and mechanical changes occurring upon external perturbation, in particular upon compression. 
Note that structural compression in ZIF-8 can be achieved by either increasing the pressure or decreasing the temperature (given that ZIF-8 shows a positive thermal expansion). ${ }^{49}$

We therefore decided to initially investigate the far-IR spectrum of ZIF-8 computationally in the terahertz region at low-pressures, corresponding to the pre-amorphization regime $(0.0-$ $0.3 \mathrm{GPa}$ ). Figure 2 shows the predicted evolution of the infrared spectrum in the terahertz region with increasing pressure. The results highlight that the most peculiar behavior on pressure in this frequency domain is indeed shown by the peak at about $2.0 \mathrm{THz}$, whose intensity increases and whose position exhibits a remarkable non-linear shift. This peak is initially blue-shifted with increasing pressure up to about $0.1 \mathrm{GPa}$, and then subsequently red-shifts when pressure is further increased. Thus, this behavior is strictly correlated to the evolution on pressure of the mechanical response of the system discussed above. It also confirms the strong link between the corresponding terahertz lattice dynamics and the structural transformations that ZIF-8 undergoes on pressure. Furthermore, the Ramanactive peak at about $1.0 \mathrm{THz}$ also exhibits the same shifting pattern (see ESI Figure S1). This confirms the clear relationship between these spectral features due to symmetric and asymmetric "swing-effect" motions of the imidazolate linkers in the four-membered rings and the mechanochemical properties of ZIF-8. A link to dynamic videos all of the vibrational modes (IR and Raman) of ZIF-8 is provided in the ESI.

We wanted to experimentally confirm the extent to which these spectral features probe the structural changes occurring in ZIF-8 upon perturbation by temperature and pressure. To do this, we have performed a novel pressure- and temperature-dependent THz-TDS experiment, using two different gases as PTM (methane and nitrogen). Figure 3 shows the results for nitrogen; Figure S2 in the ESI shows the results for methane, which are qualitatively similar. First we observe a significant absorption peak initially near $2.0 \mathrm{THz}$ in the empty ZIF-8, which we refer to as $\omega^{*}$. This peak is consistent with the previously reported standard THz-TDS result ${ }^{29}$ indicating that the sample is crystalline. In that study, this feature was observed to significantly broaden at low temperatures and exhibit an anomalous temperature- 

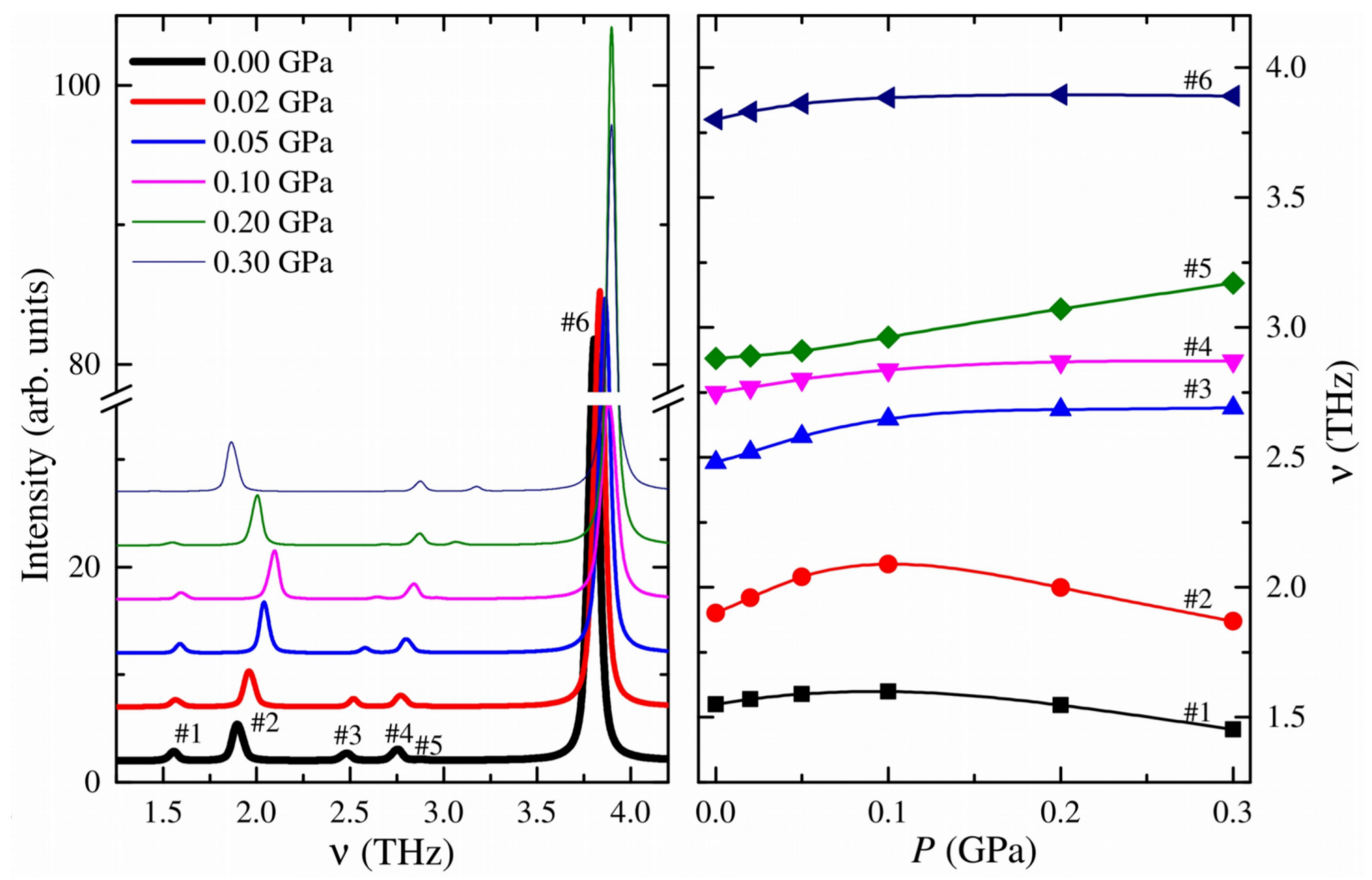

Figure 2: DFT-predicted infrared spectrum of ZIF-8 as a function of pressure $(0.0-0.30 \mathrm{GPa})$ in the terahertz region (left) and the evolution of the IR-active vibrational modes as a function of pressure (right). 

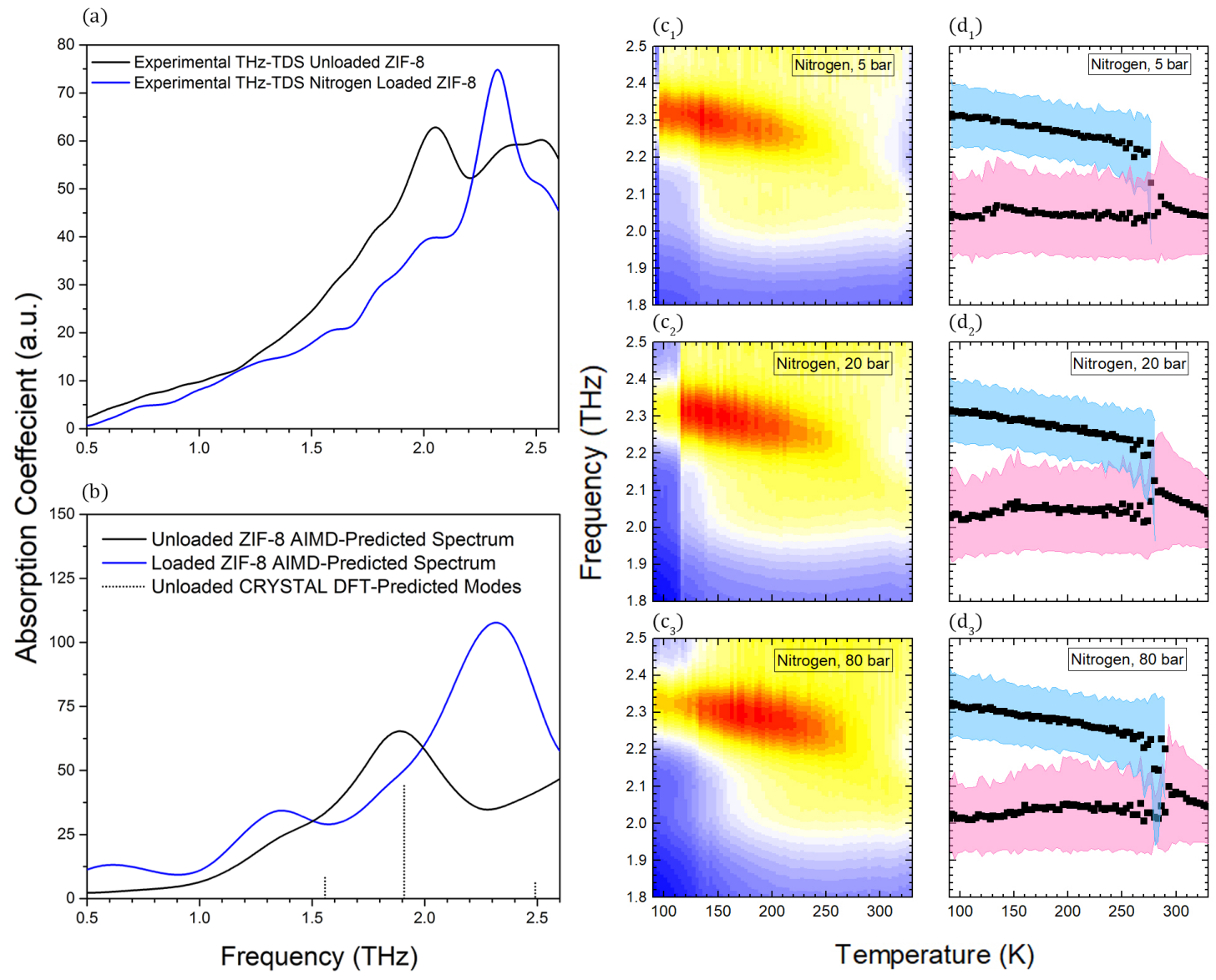

Figure 3: (a) Experimental and (b) ab initio molecular dynamics (AIMD) temperature dependent terahertz absorption spectra at $97 \mathrm{~K}$ for ZIF-8 unloaded (black) and loaded with $\mathrm{N}_{2}$ at 80 bar. The vertical black dotted lines represent the transitions predicted by CRYSTAL17 using static-DFT simulations on a pristine crystal. (c) THz-TDS spectra under varying temperature and pressure, with red and blue colors representing the maximum and minimum of the absorption intensity, respectively; the sharp transitions in $\left(\mathbf{c}_{1}\right)$ and $\left(\mathbf{c}_{2}\right)$ at $95 \mathbf{K}$ and $115 \mathbf{K}$, respectively, are caused by the change of the refractive index of the sample due to the gas-to-liquid phase transition of nitrogen. (d) Black squares are fitted peak positions while pink and blue shadows are the corresponding line-widths at half-maximum for the peaks at about $2.0 \mathrm{THz}$ and $2.3 \mathrm{THz}$, respectively. 
induced shifting. In the present study, we clearly observed that as temperature is lowered, and structural compression is induced, the frequency of $\omega^{*}$ increased. This was followed by a sudden decrease at about $290 \mathrm{~K}$ and 5 bar (Figure 3 (d)), followed by a plateau extending to about $190 \mathrm{~K}$. This feature exhibits the exact same behavior upon compression as predicted by the quantum-mechanical simulations discussed above (Figure 2), however it is important to note the range of pressures explored in Figure $2(0.1 \mathrm{GPa}=1000 \mathrm{bar})$ and those in Figure 3 (5-80 bar), with the former performed at $0 \mathrm{~K}$ and the latter incorporating thermal effects. The discontinuity of $\omega^{*}$ on evolution of temperature, reflected in the change in sign of $\partial \omega^{*} / \partial T$, shifts to higher temperatures with increasing $\mathrm{N}_{2}$ pressure, from $288 \mathrm{~K}$ at 5 bar to $297 \mathrm{~K}$ at 80 bar. Clearly, as the external pressure is increased, a lower degree of thermally-induced compression is required to achieve the same overall compression of the structure. These results highlight that the THz-TDS experiments provide a valuable probe of relevant collective structural changes in $\mathrm{ZIF}-8$, in a non-invasive and non-destructive manner.

In addition to the $2.0 \mathrm{THz}$ mode, we further noted that a new spectral peak appears at about $2.3 \mathrm{THz}$ at the exact same temperature at which the structural and mechanical features described above exhibit the discontinuity (Figure 3 a and c); Figure 3 (d)) shows this through the blue shaded regions. The origins of this spectral feature are understood in terms of an intrinsic vibrational mode of the framework that is activated by the presence of adsorbed gas molecules in the pores of the solid. Through the use of ab initio molecular dynamics simulations (AIMD) we evaluated the vibrational response of a pristine and loaded (48 nitrogen molecules per cell) ZIF-8 crystal by taking the dipole-moment autocorrelation function of a 30 ps NVT trajectory, and the results are shown in Figure 3(b). Compared to the pristine (empty) ZIF-8 crystal, an additional feature is clearly predicted in the loaded variant at about $2.3 \mathrm{THz}$. The "swing-effect" mode at $\sim 2 \mathrm{THz}$ is present in both regimes, with the loaded ZIF-8 presenting a lower intensity for that feature, as observed experimentally (see again Figure 3 (a)). We then deconvolved the spectra into contributions from the host (ZIF-8) and guest $\left(\mathrm{N}_{2}\right)$ molecules. We found that the nitrogen 
molecules did not directly contribute to the low-frequency spectrum of ZIF-8 on their own, but rather dramatically enhance the intensity of an intrinsic spectral feature by breaking a symmetric structural configuration. Indeed, there is a normal mode predicted from the solid-state DFT simulations at about $2.5 \mathrm{THz}$, which involves a symmetric bending of two imidazolate linkers around a central imidazolate, in a pincer-type motion, which is similar in frequency to both the experimental and AIMD peaks at about $2.3 \mathrm{THz}$. This mode has a low intensity for the pristine solid because crystallographic symmetry results in the net cancellation of dipole moments for the entire unit cell. However, when the system is loaded, the nitrogen molecules affect this vibration by enabling anisotropic motion through the removal of stringent crystalline symmetry, i.e. each imidazolate linker rotates with differing degrees of motion depending on the nitrogen environment, yielding a much larger net change in the dipole moment for the unit cell and thus a greater IR intensity.

\section{Elucidating the Intrinsic-Framework Nature of the High-Pressure Phase Transition with DFT Simulations}

The successful modeling of the structural, dynamical, and mechanical phenomena in the lowpressure regime lends valuable confidence to the theoretical methodology used, and enables deeper investigation into the pressure-dependent properties of ZIF-8 to be explored. The observed first-order phase transition at high pressure ${ }^{20}$ is well-accepted to be assisted by the presence of adsorbed molecules ${ }^{50}$ that are known to play a key role in preventing the amorphization of the framework, however the role that the ZIF framework alone plays remains unclear. Here, we wanted to understand whether or not the observed phase transition is an intrinsic feature of the pristine ZIF-8 framework or if the transition requires the presence of adsorbed gas molecules, all when amorphization is hindered. Experimentally, amorphization can be hindered at high-pressure by the adsorption of guest molecules in the pores, however performing experiments at high-pressure without a PTM is not possible due to the pristine framework undergoing amorphization at pressures above $0.34 \mathrm{GPa},{ }^{19}$ as previously 


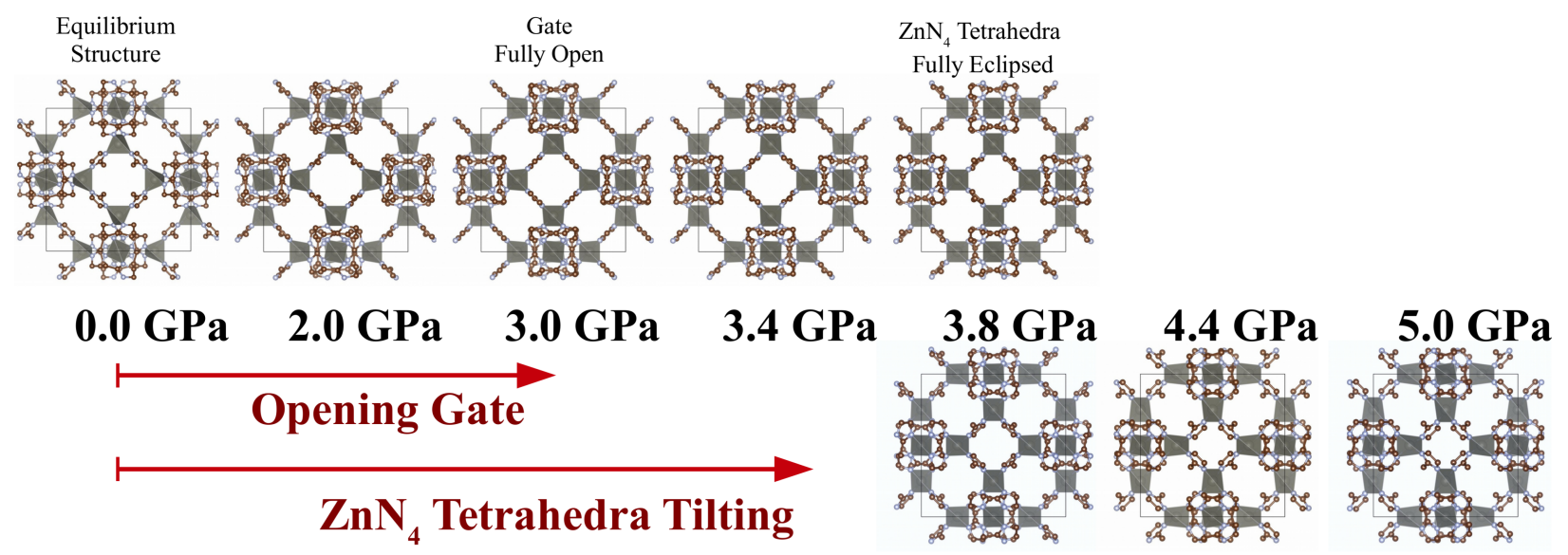

Figure 4: Pressure-induced structural changes through the first-order phase transition and up to $5 \mathrm{GPa}$ in pristine $\mathrm{ZIF}-8$.

mentioned. Thus, the inherent pressure-dependent properties of the pristine framework at high-pressures cannot be captured experimentally. With our theoretical simulations, the crystalline lattice can be preserved also at high-pressures by imposing periodic-boundary conditions, which prohibits long-range structural deviations and therefore prohibits amorphization. While this method cannot be confirmed experimentally and without question the results are purely theoretical, understanding how the application of high-pressures affects the pristine ZIF-8 framework provides valuable insight into the mechanical response of the solid in the absence of adsorbed gas molecules, an important aspect for understanding MOFs in general. In this sense, the accurate modeling of the low-pressure behavior of ZIF-8 provides some confidence that the forces within the solid are well-reproduced, and thus the data presented here can be considered accurate within the short-range limit.

We therefore wrapped up our study by investigating the pressure-induced structural changes of the pristine ZIF-8 up to 5 GPa using quantum-mechanical simulations with CRYSTAL17. Figure 4 shows the pressure-induced structural changes through the first-order phase transition while the evolution of unit cell volume, $\varphi$, and $\theta$ as a function of pressure is reported in Figure 5. We first predicted a first-order phase transition (indicated by dashed vertical red lines in Figure 5), which is clearly identified by a sharp discontinuity in all structural features, namely a $1.4 \%$ drop in unit cell volume. This is the first evidence of the 
intrinsic pressure-induced, rather than extrinsic adsorption-induced, nature of the observed first-order phase transition in ZIF-8, although experimentally probing this proves difficult due to the limitation of keeping the solid in its pristine state and avoiding amorphization.

The low-pressure and high-pressure phases of ZIF-8 exhibit the same $I \overline{4} 3 m$ space group symmetry (i.e. $I \overline{4} 3 m \rightarrow I \overline{4} 3 m$ ), as experimentally reported when using a penetrating PTM. However, the atomistic structural changes characterizing the phase transition in the pristine ZIF-8 show peculiar features, which differ significantly from those observed experimentally in the loaded system. First, the imidazolate linkers almost linearly rotate as a function of pressure. At zero pressure they are in a "gate closed" configuration with $\varphi \simeq 20^{\circ}$, then, they reach the "gate fully open" configuration with $\varphi=0^{\circ}$ at $3.8 \mathrm{GPa}$ and, as pressure further increases they continue rotating towards another "gate closed" configuration with $\varphi \simeq-20^{\circ}$. This is quite different with respect to what happens in the relative high-pressure adsorption regime where a high concentration of guest molecules in the pores constrains the linkers to stay "fully open". Secondly, in our simulations, the $\mathrm{ZnN}_{4}$ tetrahedra also tilt almost linearly as a function of pressure, which is not observed experimentally. At zero pressure $\theta \simeq 50^{\circ}$, and interestingly in our simulations the $\theta$ angle goes to zero precisely at the predicted transition pressure of 3.8 GPa. The first-order phase transition in pristine ZIF-8 thus occurs when the $\mathrm{ZnN}_{4}$ tetrahedra are fully eclipsed and the angle $\theta$ (rather than the "gate-opening" angle $\varphi$ ) is to be considered the "order parameter" describing the phase transition.

When penetrating PTM are used to convey pressure in the experiments, it is to be expected that the local pressure of the guest molecules acting on the imidazolate linkers would lead to a faster opening of the gate towards a "fully open" configuration, thus allowing more molecules in the pores through the creation of new adsorption sites. In turn, the new adsorption sites help to stabilize the "gate-open" phase. This explains the lower transition pressure observed experimentally for loaded samples with respect to the pristine system. This is further confirmed by the results of the AIMD simulations (vide supra), which indicate that larger displacements of the linkers occur upon loading. 


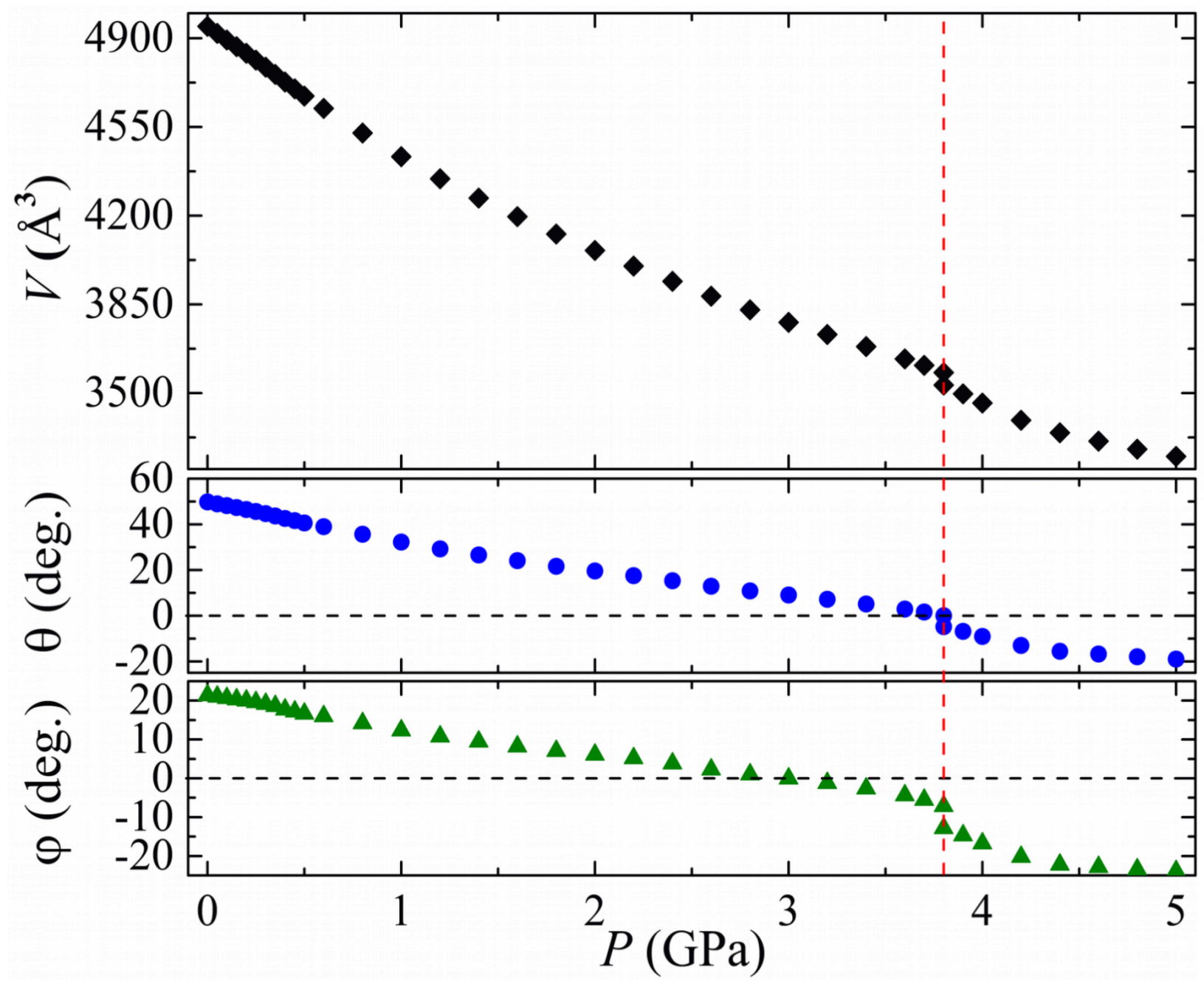

Figure 5: Volume $V$, imidazolate linkers "gate-opening" rotation angle $\varphi$, and $\mathrm{ZnN}_{4}$ tetrahedra tilting angle $\theta$ as a function of pressure. 
The results reported in Figure 5 aid in suggesting that the first-order phase transition experimentally observed at $1.47 \mathrm{GPa}$ is due to intrinsic short-ranged structural features of ZIF-8. Because the theoretical application of a hydrostatic pressure alone, with the concomitant imposition of translation symmetry, is able to capture a first order transition that resembles what is observed experimentally, this suggests that long-range effects are not as critical for the phase transition as short-range forces. However, without question the experimentally observed phenomena are different from what is observed here, with the main difference being the adsorbed gas molecules significantly influencing the short-range interactions and thus altering the observed pressure and nature of the phase transition. Regardless, the results presented here, by working within periodic boundary conditions on the pristine system, have demonstrated that the short range intrinsic structural changes are significant factors in the pressure-induced phase transition in ZIF-8.

\section{Conclusions}

A peculiar non-linear trend of the mechanochemical response of ZIF-8 as a function of pressure is reported. The bulk modulus of the pristine system increases up to $0.1 \mathrm{GPa}$ of pressure while the system becomes mechanically softer as pressure is further increased up to $0.34 \mathrm{GPa}$, before undergoing amorphization. The combination of experimental pressureand temperature-dependent THz time-domain spectroscopy (TDS) and quantum mechanical simulations allows for an atomistic characterization of the static structural changes and dynamic collective lattice vibrations responsible for this behavior. The explicit connection between symmetric and asymmetric "gate-opening" motions of the imidazolate linkers in the four-membered rings of the ZIF-8 framework and its mechanical response is reported. These

low-frequency collective vibrations are probed experimentally with THz-TDS as a function of structural compression, and a clear correlation is reported with the corresponding mechanical response on pressure. The methodology presented here can be applied to any supramolecular 
material. The short-ranged intrinsic nature of the high-pressure first-order phase transition of ZIF-8 is unveiled.

\section{Acknowledgments}

M.T.R. thanks the University of Vermont for support. M.T.R. and J.A.Z. thank the UK Engineering and Physical Sciences Research Council for funding (EP/N022769/1), and via our membership of the UK's HEC Materials Chemistry Consortium, which is funded by EPSRC (EP/L000202), this work used the ARCHER UK National Supercomputing Service (http://www.archer.ac.uk). W.Z. and D.M.M. thank the R. A. Welch Foundation for funding. A.E. and J.M. thank the University of Torino and the Compagnia di San Paolo for funding (CSTO169372). This project has received funding from the European Research Council (ERC) under the European Union's Horizon 2020 research and innovation pro-

gramme (NanoMOFdeli), ERC-2016-COG 726380. D.F.-J. thanks the Royal Society for funding through a University Research Fellowship.

\section{Supporting Information}

Pressure dependence of IR and Raman active 'swing-effect' mode, pressure dependent experimental THz-TDS data using methane as the PTM, video of the pressure dependent structural change in ZIF-8, INPUT files for CRYSTAL, the vibrational modes of ZIF-8 can be visualized on the following webpage http://www.crystal.unito.it/vibs/ZIF-8_under_ pressure/index.html?name=zif8_m062x_0.0gpa_freq.xyz

\section{References}

(1) Goodwin, A. L.; Kepert, C. J. Negative Thermal Expansion And Low-Frequency Modes in Cyanide-Dridged Framework Materials. Phys. Rev. B 2005, 71, 140301. 
(2) Zhou, W.; Wu, H.; Yildirim, T.; Simpson, J. R.; Walker, A. R. H. Origin of the Exceptional Negative Thermal Expansion in Metal-Organic Framework-5. Phys. Rev. B 2008, 78, 054114.

(3) Brozek, C. K.; Michaelis, V. K.; Ong, T.-C.; Bellarosa, L.; Lopez, N.; Griffin, R. G.; Dinca, M. Dynamic DMF Binding in MOF-5 Enables the Formation of Metastable Cobalt-Substituted MOF-5 Analogues. ACS Central Science 2015, 1, 252-260.

(4) Van Yperen-De Deyne, A.; Hendrickx, K.; Vanduyfhuys, L.; Sastre, G.; Van Der Voort, P.; Van Speybroeck, V.; Hemelsoet, K. Dynamic DMF Binding in MOF5 Enables the Formation of Metastable Cobalt-Substituted MOF-5 Analogues. Theo. Chem. Acc. 2016, 135, 102.

(5) Turton, D. A.; Senn, H. M.; Harwood, T.; Lapthorn, A. J.; Ellis, E. M.; Wynne, K. Terahertz Underdamped Vibrational Motion Governs Protein-Ligand Binding in Solution. Nat. Commun. 2014, 5, 028103.

(6) Gerstman, B.; Roberson, M.; Austin, R. Enhancement of Ligand Binding to Myoglobin By Far-Infrared Excitation From a Free-Electron Laser. JOSA B 1989, 6, 1050-1057.

(7) Ruggiero, M. T.; Krynski, M.; Kissi, E. O.; Sibik, J.; Markl, D.; Tan, N. Y.; Arslanov, D.; Van Der Zande, W.; Redlich, B.; Korter, T. M. et al. The Significance of the Amorphous Potential Energy Landscape For Dictating Glassy Dynamics and Driving Solid-State Crystallisation. Phys. Chem. Chem. Phys. 2017, 19, 30039-30047.

(8) Ryder, M. R.; Civalleri, B.; Cinque, G.; Tan, J.-C. Discovering Connections Between Terahertz Vibrations And Elasticity Underpinning the Collective Dynamics of the. CrystEngComm 2016, 18, 4303-4312.

(9) Ryder, M. R.; Civalleri, B.; Bennett, T. D.; Henke, S.; Rudić, S.; Cinque, G.; Fernandezalonso, F.; Tan, J.-C. Identifying the Role of Terahertz Vibrations in Metal-Organic 
Frameworks: From Gate-Opening Phenomenon to Shear-Driven Structural Destabilization. Phys. Rev. Lett. 2014, 113, 215502.

(10) Mason, J. A.; Oktawiec, J.; Taylor, M. K.; Hudson, M. R.; Rodriguez, J.; Bachman, J. E.; Gonzalez, M. I.; Cervellino, A.; Guagliardi, A.; Brown, C. M. et al. Methane Storage in Flexible Metal-Organic Frameworks with Intrinsic Thermal Management. Nat. Commun. 2015, 527, 357-361.

(11) Zhuang, J.; Kuo, C.-H.; Chou, L.-Y.; Liu, D.-Y.; Weerapana, E.; Tsung, C.-K. Optimized Metal-Organic-framework Nanospheres For Drug Delivery: Evaluation of Smallmolecule Encapsulation. ACS Nano 2014, 8, 2812-2819.

(12) Rogge, S. M. J.; Bavykina, A.; Hajek, J.; Garcia, H.; Olivos-Suarez, A. I.; SepúlvedaEscribano, A.; Vimont, A.; Clet, G.; Bazin, P.; Kapteijn, F. et al. Metal-Organic And Covalent Organic Frameworks As Single-Site Catalysts. Chem. Soc. Rev. 2017, 46, 3134-3184.

(13) Miralda, C. M.; Macias, E. E.; Zhu, M.; Ratnasamy, P.; Carreon, M. A. Zeolitic Imidazole Framework-8 Catalysts in the Conversion of $\mathrm{Co}_{2}$ to Chloropropene Carbonate. ACS Catal. 2012, 2, 180-183.

(14) Koyuturk, B.; Altintas, C.; Kinik, F. P.; Keskin, S.; Uzun, A. Improving Gas Separation Performance of ZIF-8 by $\left.\left[\mathrm{BmIm}_{[}\right] \mathrm{BF}_{4}\right]$ Incorporation: Interactions and Their Consequences on Performance. J. Phys. Chem. C 2017, 121, 10370-10381.

(15) Furukawa, H.; Cordova, K. E.; O’Keeffe, M.; Yaghi, O. M. The Chemistry And Applications of Metal-Organic Frameworks. Science 2013, 341, 1230444-1230444.

(16) Moghadam, P. Z.; Li, A.; Wiggin, S. B.; Tao, A.; Maloney, A. G. P.; Wood, P. a.; Ward, S. C.; Fairen-Jimenez, D. Development of a Cambridge Structural Database Subset: A Collection of Metal-Organic Frameworks for Past, Present, and Future. Chem. Mater. 2017, 29, 2618-2625. 
(17) Wilmer, C. E.; Leaf, M.; Lee, C. Y.; Farha, O. K.; Hauser, B. G.; Hupp, J. T.; Snurr, R. Q. Large-Scale Screening of Hypothetical Metal-Organic Frameworks. Nat. Chem. 2012, 4, 83-89.

(18) Bux, H.; Liang, F.; Li, Y.; Cravillon, J.; Wiebcke, M.; Caro, J. Zeolitic Imidazolate Framework Membrane with Molecular Sieving Properties By Microwave-assisted Solvothermal Synthesis. J. Am. Chem. Soc. 2009, 131, 16000-16001.

(19) Chapman, K. W.; Halder, G. J.; Chupas, P. J. Pressure-induced Amorphization And Porosity Modification in a Metal-Organic Framework. J. Am. Chem. Soc. 2009, 131, $17546-17547$.

(20) Moggach, S. A.; Bennett, T. D.; Cheetham, A. K. The Effect of Pressure on ZIF-8: Increasing Pore Size with Pressure and the Formation of a High-Pressure Phase at 1.47 GPa. Angew. Chem. Int. Ed. 2009, 48, 7087-7089.

(21) Fairen-Jimenez, D.; Moggach, S. A.; Wharmby, M. T.; Wright, P. A.; Parsons, S.; Duren, T. Opening the Gate: Framework Flexibility in ZIF-8 Explored by Experiments and Simulations. J. Am. Chem. Soc. 2011, 133, 8900-8902.

(22) Zhang, L.; Hu, Z.; Jiang, J. Sorption-induced Structural Transition of Zeolitic Imidazolate Framework-8: A Hybrid Molecular Simulation Study. J. Am. Chem. Soc. 2013, $135,3722-3728$.

(23) Im, J.; Yim, N.; Kim, J.; Vogt, T.; Lee, Y. High-pressure Chemistry of a Zeolitic Imidazolate Framework Compound in the Presence of Different Fluids. J. Am. Chem. Soc. 2016, 138, 11477-11480.

(24) Ortiz, A. U.; Boutin, A.; Fuchs, A. H.; Coudert, F.-X. Investigating the PressureInduced Amorphization of Zeolitic Imidazolate Framework. J. Phys. Chem. Lett. 2013, 4, 1861-1865. 
(25) Hobday, C. L.; Woodall, C. H.; Lennox, M. J.; Frost, M.; Kamenev, K.; Düren, T.; Morrison, C. A.; Moggach, S. A. Understanding the Adsorption Process in ZIF-8 Using High Pressure Crystallography And Computational Modelling. Nat. Commun. 2018, 9, 1429.

(26) Casco, M. E.; Cheng, Y. Q.; Daemen, L. L.; Fairen-Jimenez, D.; RamosFernandez, E. V.; Ramirez-Cuesta, A. J.; Silvestre-Albero, J. Gate-opening Effect in ZIF-8: The First Experimental Proof Using Inelastic Neutron Scattering. Chem. Commun. 2016, 52, 3639-3642.

(27) Coudert, F.-X. Molecular Mechanism of Swing Effect in Zeolitic Imidazolate Framework. ChemPhysChem 2017, 18, 2732-2738.

(28) Ruggiero, M. T.; Bardon, T.; Strlič, M.; Taday, P. F.; Korter, T. M. The Role of Terahertz Polariton Absorption in the Characterization of Crystalline Iron Sulfate Hydrates. Phys. Chem. Chem. Phys. 2015, 17, 9326-9334.

(29) Tan, N. Y.; Ruggiero, M. T.; Orellana-Tavra, C.; Tian, T.; Bond, A. D.; Korter, T. M.; Fairen-Jimenez, D.; Zeitler, J. A. Investigation of the Terahertz Vibrational Modes of ZIF-8 and ZIF-90 with Terahertz Time-Domain Spectroscopy. Chem. Commun. 2015, 51, 16037-16040.

(30) Tanno, T.; Watanabe, Y.; Umeno, K.; Matsuoka, A.; Matsumura, H.; Odaka, M.; Ogawa, N. In Situ Observation of Gas Adsorption onto ZIF-8 Using Terahertz Waves. J. Phys. Chem. C 2017, 121, 17921-17924.

(31) Parrott, E. P. J.; Zeitler, J. A. Terahertz Time-Domain And Low-frequency Raman Spectroscopy of Organic Materials. Appl. Spectrosc. 2015, 69, 1-25.

(32) Elcombe, M. M.; Pryor, A. W. The Lattice Dynamics of Calcium Fluoride. J. Phys. C 1970, 3, 492-499. 
(33) Freire, J. D.; Katiyar, R. S. Lattice Dynamics of Crystals with Tetragonal Batio3structure. Phys. Rev. B 1988, 37, 2074-2085.

(34) Dovesi, R.; Erba, A.; Orlando, R.; Zicovich Wilson, C. M.; Civalleri, B.; Maschio, L.; Rérat, M.; Casassa, S.; Baima, J.; Salustro, S. et al. Quantum-Mechanical Condensed Matter Simulations with CRYstal. WIREs Comput Mol Sci 2018, 110, e1360.

(35) Zhao, Y.; Truhlar, D. G. The M06 Suite of Density Functionals for Main Group Thermochemistry, Thermochemical Kinetics, Noncovalent Interactions, Excited States, and Transition Elements: Two New Functionals and Systematic Testing of Four M06-Class Functionals and 12 Other Functionals. Theor. Chem. Acc. 2007, 120, 215-241.

(36) Zhu, A.-X.; Lin, R.-B.; Qi, X.-L.; Liu, Y.; Lin, Y.-Y.; Zhang, J.-P.; Chen, X.-M. Zeolitic Metal Azolate Frameworks (MAFs) from $\mathrm{ZnO} / \mathrm{Zn}(\mathrm{OH})_{2}$ and Monoalkyl-Substituted Imidazoles and 1,2,4-triazoles: Efficient Syntheses and Properties. Microporous Mesoporous Mater. 2012, 157, 42-49.

(37) Erba, A.; Mahmoud, A.; Belmonte, D.; Dovesi, R. High Pressure Elastic Properties of Minerals From Ab Initio Simulations: The Case of Pyrope, Grossular And Andradite Silicate Garnets. J. Chem. Phys. 2014, 140, 124703.

(38) Zicovich-Wilson, C. M.; Pascale, F.; Roetti, C.; Saunders, V. R.; Orlando, R.; Dovesi, R. Calculation of the Vibration Frequencies of Alpha-Quartz: the Effect of Hamiltonian And Basis Set. J. Comput. Chem. 2004, 25, 1873-1881.

(39) Hutter, J.; Iannuzzi, M.; Schiffmann, F.; Vandevondele, J. Cp2k: Atomistic Simulations of Condensed Matter Systems. WIREs Comput Mol Sci 2013, 4, 15-25.

(40) Nosé, S. A Unified Formulation of the Constant Temperature Molecular Dynamics Methods. J. Chem. Phys. 1998, 81, 511-519. 
(41) Perdew, J.; Burke, K.; Ernzerhof, M. Generalized Gradient Approximation Made Simple. Phys. Rev. Lett. 1996, 77, 3865-3868.

(42) Vandevondele, J.; Hutter, J. Gaussian Basis Sets For Accurate Calculations On Molecular Systems in Gas And Condensed Phases. J. Chem. Phys. 2007, 127, 114105.

(43) Goedecker, S.; Teter, M.; Hutter, J. Separable Dual-space Gaussian Pseudopotentials. Phys. Rev. B 1996, 54, 1703.

(44) Grimme, S.; Antony, J.; Ehrlich, S.; Krieg, H. A Consistent And Accurate ab initio Parametrization of Density Functional Dispersion Correction. J. Chem. Phys. 2010, 132, 154104.

(45) Brehm, M.; Kirchner, B. TRAVIS - a Free Analyzer and Visualizer for Monte Carlo and Molecular Dynamics Trajectories. J. Chem. Inf. Model. 2011, 51, 2007-2023.

(46) Ruggiero, M. T.; Zeitler, J. A. Resolving the Origins of Crystalline Anharmonicity Using Terahertz Time-domain Spectroscopy and ab initio Simulations. J. Phys. Chem. B 2016, 120, 11733-11739.

(47) Zhang, W.; Nickel, D.; Mittleman, D. High-pressure Cell For Terahertz Time-Domain Spectroscopy. Opt. Express 2017, 25, 2983-2993.

(48) Tan, J.-C.; Civalleri, B.; Lin, C.-C.; Valenzano, L.; Galvelis, R.; Chen, P.-F.; Bennett, T. D.; Mellot-Draznieks, C.; Zicovich-Wilson, C. M.; Cheetham, A. K. Exceptionally Low Shear Modulus in a Prototypical Imidazole-based Metal-Organic Framework. Phys. Rev. Lett. 2012, 108, 095502.

(49) Bourg, L. B.; Ortiz, A., A. U.and Boutin; Coudert, F.-X. Thermal And Mechanical Stability of Zeolitic Imidazolate Frameworks Polymorphs. APL Mater. 2014, 2, 124110.

(50) Fairen-Jimenez, D.; Galvelis, R.; Torrisi, A.; Gellan, A. D.; Wharmby, M. T.; Wright, P. A.; Mellot Draznieks, C.; Düren, T. Flexibility and Swing Effect on the 
Adsorption of Energy-related Gases on ZIF-8: Combined Experimental and Simulation Study. Dalton Trans. 2012, 41, 10752-10762. 